\title{
Length-dependent extraction of single-walled carbon nanotubes
}

\author{
Kirk J. Ziegler ${ }^{\dagger}$, Daniel J. Schmidt, Urs Rauwald, Kunal N. Shah, Erica L. Flor, \\ Robert H. Hauge, and Richard E. Smalley ${ }^{*}$ \\ Department of Chemistry, Center for Nanoscale Science and Technology, \\ Rice University, Houston, Texas 77005, USA
}

* Corresponding author: Tel: 713-348-3250; Fax: 713-348-5320; email: res@rice.edu

${ }^{\dagger}$ Current address: Department of Chemical Engineering, University of Florida, Gainesville, Florida 32611, USA

\section{Length measurements}

For length analysis, only a fraction of the organic layer was collected for analysis. These solutions were diluted and washed with acetic acid, filtered, and then re-suspended in methanol. The solutions were diluted visually to obtain the correct color of solution that is known to give adequate AFM coverage for image analysis. These suspensions can then be spin-coated onto freshly cleaved mica substrates after brief, mild sonication ( 1 min) yielding a high quantity of individual nanotubes as seen in the AFM images. Due to difficulties in controlling the exact concentration of the samples, some samples are more dilute as seen in some AFM images.

To eliminate the tedious nature of manually measuring $1500+$ nanotubes, the lengths are measured using the Nanotube Length Analysis module of the SIMAGIS (Smart Imaging Technologies, Houston, Texas) software package. This software package has the capability of automatically analyzing multiple AFM images to obtain statistically accurate length and height (diameter) measurements of the nanotubes contained in the sample. Some images may appear to vary from the average length measured because there is a distribution of lengths and each image will not yield the same average length. This factor becomes especially important for images taken at lower concentrations. For this reason, multiple AFM images are collected (typically $6-12$ ) so that numerous SWNTs can be measured. The error bars given in Figure 6 help to demonstrate how much variance could be seen in the average length of a given image. The maximum and minimum error bars were taken as the lowest and highest average achieved for an individual image. For example, the measurement at $40 \%$ ion-pairing had one image that gave an average of $80 \mathrm{~nm}$ while another image gave an average of 135 $\mathrm{nm}$. However, the collection of all images (16 images) gave an average length of 109 nm. The measurements were demonstrated to be reproducible, i.e. repeating the preparation of AFM samples and images yielded similar length measurements. 

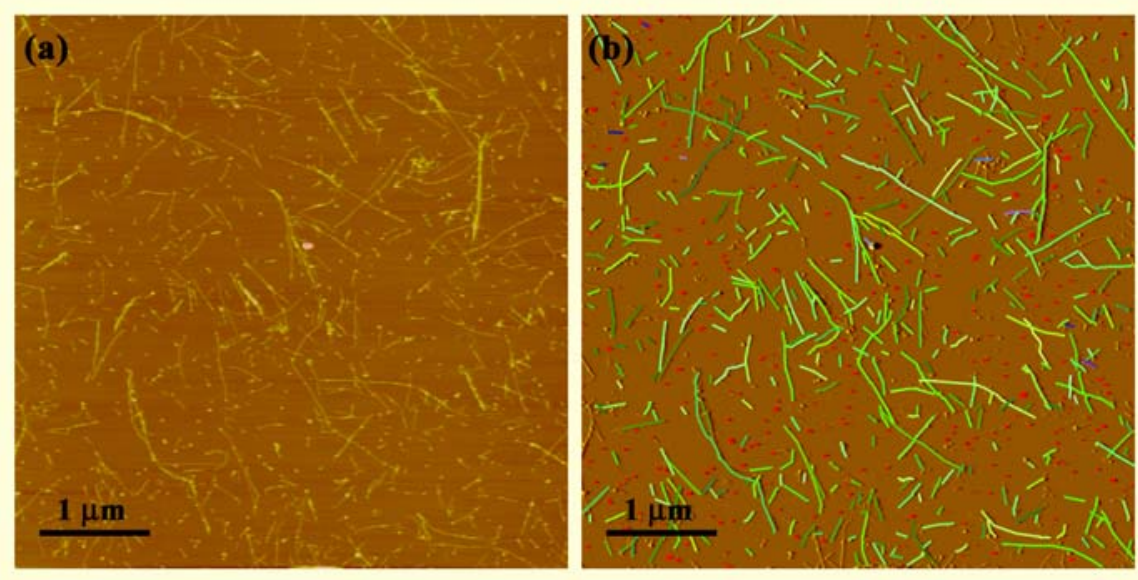

Figure S1. (a) AFM image of the functionalized SWNTs before extraction. (b) Nanotube trace determined by SIMAGIS software. 

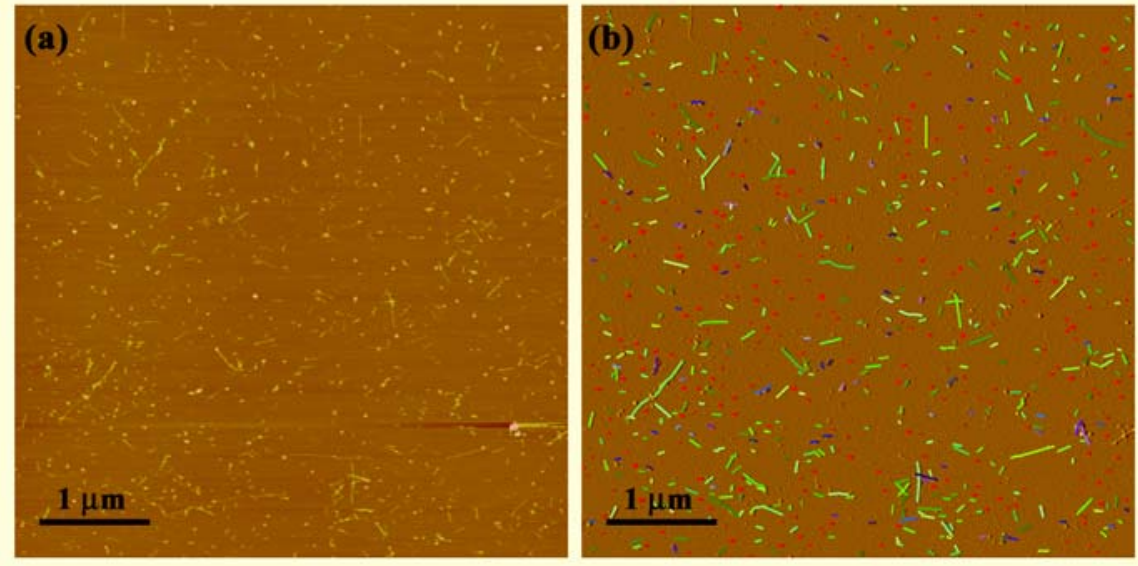

Figure S2. (a) AFM image of the functionalized SWNTs after partial extraction with TOAB corresponding to ion-pairing of $30 \%$. (b) Nanotube trace determined by SIMAGIS software. 

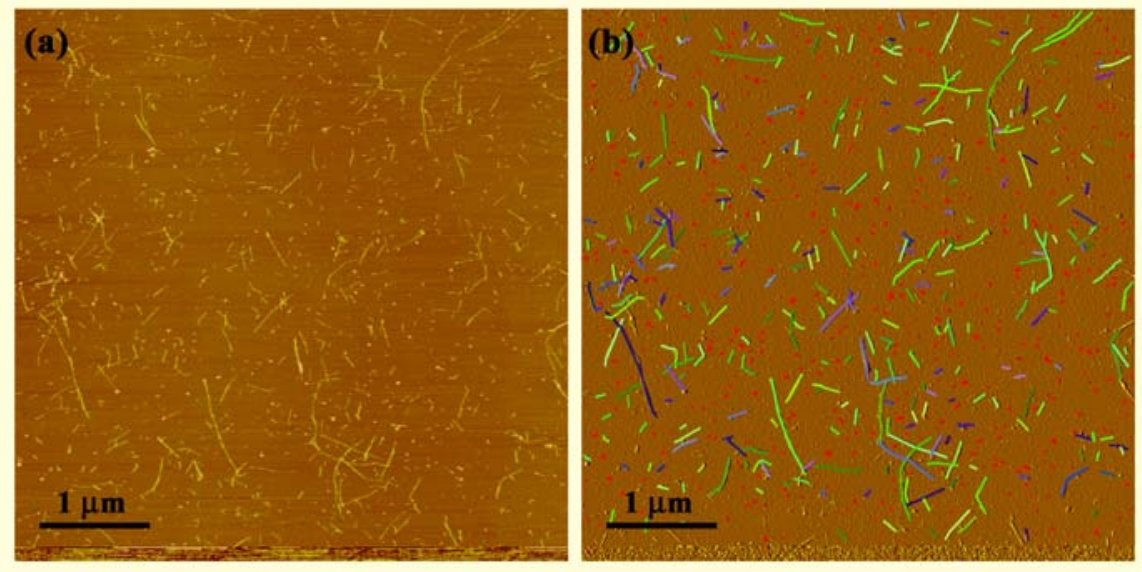

Figure S3. (a) AFM image of the functionalized SWNTs after partial extraction with TOAB corresponding to ion-pairing of $60 \%$. (b) Nanotube trace determined by SIMAGIS software. 

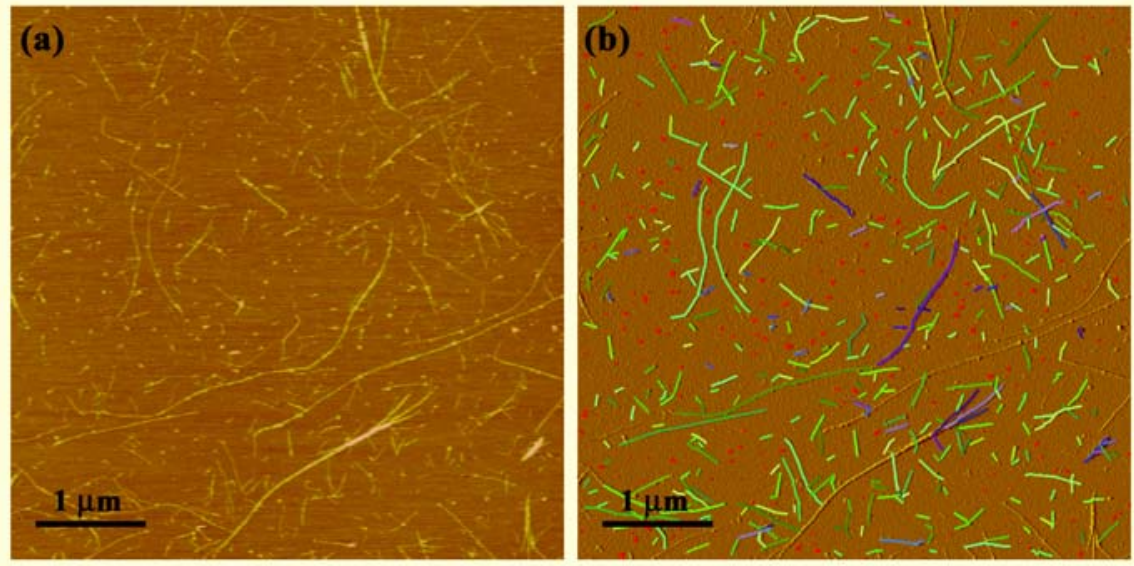

Figure S4. (a) AFM image of the functionalized SWNTs after partial extraction with TOAB corresponding to ion-pairing of $75 \%$. (b) Nanotube trace determined by SIMAGIS software. 